\title{
Kernos
}

Revue internationale et pluridisciplinaire de religion grecque antique

$15 \mid 2002$

Varia

\section{Antoine HERMARY, Amathonte V. Les figurines en terre cuite archaïques et classiques. Les sculptures en pierre}

Thierry Petit

\section{(2)enEdition}

\section{Journals}

Édition électronique

URL : http://journals.openedition.org/kernos/1449

DOI : 10.4000/kernos. 1449

ISSN : 2034-7871

\section{Éditeur}

Centre international d'étude de la religion grecque antique

\section{Édition imprimée}

Date de publication : 1 janvier 2002

ISSN : 0776-3824

\section{Référence électronique}

Thierry Petit, « Antoine heRmARY, Amathonte V. Les figurines en terre cuite archaïques et classiques. Les sculptures en pierre », Kernos [En ligne], 15 | 2002, mis en ligne le 16 juin 2011, consulté le 24 septembre 2020. URL : http://journals.openedition.org/kernos/1449 ; DOI : https://doi.org/10.4000/ kernos. 1449 
encore une synthèse; sans conteste $V$. Karageorghis était le mieux à même de la mener à bien. C'est désormais chose faite.

Thierry Petit

(Université de Strasbourg II)

Antoine Hermary, Amatbonte $V$. Les figurines en terre cuite archaiques et classiques. Les sculptures en pierre, Paris, École française d'Athènes / Fondation A. Leventis, 2000. 1 vol. $29,7 \times 20,9 \mathrm{~cm}, 187$ p., 95 pl. (Études chypriotes, 15).

Faisant suite au volume Amatbonte II. Testimonia 2 : La sculpture, de 1981, ce livre traite de l'ensemble des trouvailles effectuées sur le sites de la ville d'Amathonte depuis deux décennies. Trois chantiers ouverts sur l'acropole de la ville antique ont livré l'essentiel des objets présentés ici. C'est d'abord l'Aphrodision, sur le sommet de la colline, dont la publication devrait rapidement voir le jour; à ces trouvailles, il faut associer celles de la terrasse ouest, située en contrebas, puisque les objets qui y furent découverts proviennent vraisemblablement du sanctuaire lui-même; enfin le palais, qui, quoique très partiellement fouillé, a livré à lui seul près de $20 \%$ des objets présentés et surtout près de la moitié des sculptures en pierre (calcaire). Le choix de regrouper en un même volume la coroplathie et la statuaire de différents chantiers présente l'inconvénient d'isoler ainsi ce matériel du contexte archéologique et des autres trouvailles. Ce désagrément aurait pu être compensé par le regroupement des objets selon leur lieu de découverte, ce qui, du moins, aurait offert un panorama cohé rent de l'environnement iconographique des différents sanctuaires d'où le matériel est issu. Tel n'a pas été le choix de l'A. qui fournit un catalogue strictement techno-typologique. Les chapitres introductif et conclusif ne compensent que partiellement cet inconvénient. Le catalogue compte 755 figurines ou fragments de figurines en terre cuite, et 277 sculptures en pierre, soit plus de mille objets. Toute l'iconographie connue dans la plastique cypriote est représentée, à l'exception notable de la grande sculpture en terre cuite. Les différentes techniques connues pour la coroplathie sont mises en œuvre. Certains types sont plus spécifiques à Amathonte, en particulier des modèles de bateaux ( $\left.\mathrm{n}^{\circ} 270-284\right)$, des groupes d'un chien attaquant un lièvre $\left(n^{\circ}\right.$ 953-954) et des masques votifs miniatures ( $n^{\circ}$ 506-527). On trouve aussi en abondance des «plaquettes d'Astarté » $\left(n^{\circ} 532-594\right)$, et des korès drapées à la grecque $\left(n^{\circ} 596-688\right)$. On s'étonnera cependant de voir figurer dans un tel catalogue des appliques ou fragments de vases plastiques $\left(n^{\circ} 729-734\right)$. La sculpture en pierre est aussi très diverse : korès à la fleur ( $n^{\circ} 787-803$ ), joueuses de tambourin ( $\left.n^{\circ} 827-827\right)$, cavaliers (uniquement au palais : $\mathrm{n}^{\circ}$ 851-854), et même un modèle de harmamaxa, ou char couvert de type perse (également du palais : $\mathrm{n}^{\circ} 868$ ), des temple-boys $\left(\mathrm{n}^{\circ} 870-873\right)$, des sphinx-tbymiatéria $\left(\mathrm{n}^{\circ} 882-926\right)$, des oiseaux ( $\mathrm{n}^{\circ}$ 948-951), etc.

S'agissant des conclusions tirées sur la topographie religieuse de l'acropole d'Amathonte, plusieurs remarques rapides peuvent être formulées. On a dit que la zone du palais ( Chantier $C$ ») et celle de la porte du rempart ( Chantier B »), quelque dizaines de mètres plus au sud, avaient livré une grande quantité de figurines et surtout de sculptures en calcaire. L'A. distingue cependant les deux chantiers et, arguant de la présence exclusive de la grande plastique dans la dernière de ces zones, suppose que les trouvailles qui y furent faites n'ont pas été entraînées par la pente, du palais vers la muraille, mais proviennent bien de la zone même où elles ont été découvertes; ce qui l'amène à supposer l'existence, comme à Vouni, d'un sanctuaire principal situé en contrebas du palais et lui donnant accès (p. 164-167). À mes yeux, pareille hypothèse se heurte à plusieurs 
difficultés. La principale étant que le grand chapiteau hathorique publié dès 1985 par A. Hermary, qu'il estime appartenir à cet hypothétique sanctuaire, provient d'une zone qui a depuis longtemps été identifiée comme appartenant au palais lui-même ( $B C H, 107,1983$, p. 967, fig. 14). Ensuite l'A. dénie tout caractère votif à certains types que l'on rencontre dans la petite plastique qui fut trouvée dans le remblai couvrant les magasins du palais; pour lui, ils constituent une « partie du mobilier de la vie quotidienne [qui] rappelait les principales activités des maîtres du lieu ». Pareille hypothèse ne laisse pas d'étonner : tout d'abord, ces nombreux objets ne peuvent provenir que de sanctuaires que l'on peut imaginer, à l'exemple de Vouni, sis tout autour du palais, voire dans le corps de l'édifice lui-même. Deux de ces ensembles cultuels palatiaux sont d'ailleurs présentés dans l'introduction de l'ouvrage (p. 5-6). Ensuite, l'interprétation de l'A. peut à la rigueur expliquer la fonction de ces artefacts, mais laisse dans l'ombre leur nature. Sauf à imaginer ces « maîtres des lieux » comme dotés d'une complexion particulièrement ludique, qu'en faire d'autre sinon des paraphernalia et des ex-voto de cultes palatiaux?

Dans un chapitre conclusif très convaincant (p. 167-169), l'A. confronte les enseignements que l'on peut tirer de ce matériel pour le culte d'Aphrodite au sanctuaire sommital avec les textes littéraires et les nombreuses études qu'il a luimême déjà consacrées au sujet.

Thierry Petit

(Université de Strasbourg II)

Maria Luisa Nava, Massimo Osanna (éds), Rituali per una Dea Lucana. Il santuario di Torre di Satriano, Università degli studi della Basilicata, Soprintendenza Archeologica della Basilicata, 2001. 1 vol, $22 \times 28 \mathrm{~cm}, 150$ p., nbr. ill. (catalogue d'exposition).

Le site de Torre di Satriano situé au sud-ouest de Potenza (Basilicate) a fait l'objet, en 2000, de nouveaux sondages archéologiques entrepris conjointement par la surintendance (M.L. Nava) et l'Université de Basilicate (sous la direction de M. Osanna). Les fouilles qui ont porté sur le sanctuaire lucanien découvert en 1987/88 par l'équipe de E. Greco ( $c f$. Satriano 1987/88. Un biennio di ricerche archeologiche, Naples, 1988) au sud-ouest de la zone urbaine complètent ainsi les données topographiques du site rassemblées une première fois en 1970 par R.R. Holloway ( $c f$. Satrianum. The Archaeological Investigations Conducted by Brown University in 1966 and 1967, Providence, 1970). Ce sont les résultats de ces nouvelles investigations qui sont publiées dans le présent ouvrage réalisé à l'occasion de l'exposition Rituali per una dea lucana organisée au musée provincial de Potenza et faisant suite à deux autres manifestations intitulées L'arcbeologia in Basilicata et Archeologia dell'acqua in Basilicata. Ce catalogue est composé de neuf chapitres rédigés par une quinzaine d'auteurs et parcourant les différentes phases de fréquentation du site depuis le Bronze Moyen jusqu'au Moyen Âge, en accordant toutefois une plus grande importance à la période lucanienne ( $\mathrm{V}^{\mathrm{e}}-\mathrm{III} \mathrm{e}^{\mathrm{e}} \mathrm{s} . \mathrm{av} . \mathrm{J} . \mathrm{C}$.). Dans le premier chapitre (Un territorio dell'Ttalia antica par M. Osanna et A. Russo, p. 13-16) les A. présentent les cadres géographiques et humains de la Lucanie antique (Basilicate et partie méridionale de la Campanie actuelle). Le chapitre suivant (Prima del Lucani par M. Osanna et M.M. Sica, p. 1728) est consacré à la description du site de Torre di Satriano et de son environnement géographique (fleuves, sources, etc.) ainsi qu'aux populations qui ont occupé l'endroit depuis le Bronze Moyen jusqu'au ves. av. J.-C. Dans le troisième chapitre (I Lucani par M. Osanna, p. 29-32), l'A. met en évidence les différents changements que l'archéologie permet d'observer dans cette région entre le $\mathrm{v}^{\mathrm{e}}$ et 